\title{
Spectrally Flat Supercontinuum Generation in a ZBLAN Fiber Pumped by Erbium-Doped Mode-Locked Fiber Laser
}

\author{
Huanhuan LIU, Ye YU, Wei SONG, Qiao JIANG, Fufei PANG*, and Tingyun WANG \\ Key Laboratory of Specialty Fiber Optics and Optical Access Networks, Joint International Research Laboratory of \\ Specialty Fiber Optics and Advanced Communication, Shanghai Institute for Advanced Communication and Data \\ Science, Shanghai University, Shanghai 200444, China \\ "Corresponding author: Fufei PANGＥ-mail: ffpang@shu.edu.cn
}

\begin{abstract}
We have experimentally demonstrated the flat supercontinuum (SC) generation using a 10-m-long $\mathrm{ZrF}_{4}-\mathrm{BaF}_{2}-\mathrm{LaF}_{3}-\mathrm{AlF}_{3}-\mathrm{NaF}$ (ZBLAN) fluoride fiber pumped by an erbium-doped mode-locked fiber laser incorporating carbon-nanotube-based saturable absorbers. In order to improve the spectral flatness of SC, the standardized single-mode fiber with different lengths is connected to the output of the mode-locked fiber laser before the pulse amplification. The generated SC with ZBLAN fiber exhibits the best spectral flatness with fluctuation less than $1.29 \mathrm{~dB}$ over the wavelength of $1571.8 \mathrm{~nm}-1803.1 \mathrm{~nm}$, showing potential applications in optical sensing.
\end{abstract}

Keywords: Flat supercontinuum; ZBLAN; mode-locked fiber laser

Citation: Huanhuan LIU, Ye YU, Wei SONG, Qiao JIANG, Fufei PANG, and Tingyun WANG, "Spectrally Flat Supercontinuum Generation in a ZBLAN Fiber Pumped by Erbium-Doped Mode-Locked Fiber Laser," Photonic Sensors, 2019, 9(4): 302-308.

\section{Introduction}

Supercontinuum (SC) laser sources with the ability to exhibit excellent wide bandwidth and good beam quality have attracted a significant scientific interest because of their wide applications covering the fields of metrology [1], molecular fingerprinting [2], infrared spectroscopy [3], as well as biomedicine [4]. SC sources also have potential applications in various long-distance optical sensing due to good beam profiles and high spectral stability [5]. Since Stolen and Lin observed SC generation in an optical fiber for the first time [6], various types of SC sources have been extensively investigated. Silica fibers have been the predominant nonlinear media of SC generation in the past decades due to their stability and low transmission loss in the visible and near-infrared regions [7]. However, using silica fibers as the nonlinear medium of SC generation is affected by the strong intrinsic material losses in the long wavelength, which limits the spectral broadening towards mid-infrared (mid-IR) [2].

Compared with silica glass fibers, soft-glass fibers have the lower transmission loss, higher damage threshold, and better environmental stability in the mid-IR [8-9]. Among all soft glass fibers, fluoride fibers and chalcogenide fibers are more technically mature and commercially available [10]. Generally, pumping the fibers close to their zero dispersion wavelengths (ZDWs) can obtain broadband SC effectively [11]. However, the ZDWs of chalcogenide fibers are usually above $4.5 \mu \mathrm{m}$ [12],

Received: 28 November 2018 / Revised: 23 March 2019

(C) The Author(s) 2019. This article is published with open access at Springerlink.com DIO:10.1007/s13320-019-0552-y

Article type: Regular 
making them difficult to find a suitable pump source to further broaden their spectrum. In contrast, fluoride glass fibers have higher damage threshold than chalcogenide fibers, which make them be ideal candidates for mid-IR SC generation, especially, $\mathrm{ZrF}_{4}-\mathrm{BaF}_{2}-\mathrm{LaF}_{3}-\mathrm{AlF}_{3}-\mathrm{NaF}$ (ZBLAN) fibers [10, 13]. So far, the wavelength range generated by ZBLAN fibers have been achieved from the ultraviolet to the mid-infrared $[14,15]$. In practical applications, the SC source produces a flat spectrum over a wide range of wavelength, which not only satisfies the system's spectral bandwidth requirements, but also improves the measurement accuracy and reduces the technical difficulty of power balance [16]. However, SC flatness, which is important for many applications, is still a challenge [7]. At present, the fluctuation of the spectrum is almost larger than $3 \mathrm{~dB}$, especially in the wavelength range of $1600 \mathrm{~nm}$ - $1800 \mathrm{~nm}$. On the other hand, SC laser sources obtained in this range have potential applications in optimization of deeper layers biological tissue imaging, especially examining cholesterol and collagen contents in different tissues in the short-wave infrared region [17]. Therefore, the spectral performances of SC laser sources are expected to be improved.

In this work, we have demonstrated flat SC generation using a 10-m-long ZBLAN fiber pumped by a $1.5-\mu \mathrm{m}$ mode-locked fiber laser incorporating carbon-nanotube-based saturable absorbers (CNTSAs). The standardized single-mode fiber (SMF) with different access lengths is connected to the output of the mode-locked fiber laser in order to improve the spectral flatness of SC. For the first time, the high spectral flatness with fluctuation less than $1.29 \mathrm{~dB}$ ranging from $1571.8 \mathrm{~nm}$ to $1803.1 \mathrm{~nm}$ has been demonstrated by using the ZBLAN fiber. The experimental observations indicate that the optimization of the initial pulse width and peak power can contribute to the generation of flat SC.

\section{Experimental setup}

Figure 1 exhibits the experimental schematic diagram of flat SC generation using the ZBLAN fiber. An erbium-doped mode-locked fiber laser incorporating a CNT-SA operating at $1550 \mathrm{~nm}$ is utilized as the seed source. The CNT-SA is fabricated by the optical-driven deposition method and plays the role of mode-locker. A $\sim 55$-cm-long erbium-doped fiber (EDF) is applied as the gain fiber which is pumped by a $980-n m$ pump laser via a 980/1550 $\mathrm{nm}$ wavelength division multiplexing (WDM) coupler. A polarization-insensitive optical isolator is applied to prevent backward propagation of the light in the cavity. The 80/20 coupler extracts $20 \%$ of the optical power from the laser cavity as the laser output. The total length of the laser cavity is $11 \mathrm{~m}$. A piece of the commercially standardized SMF outside the laser cavity is used to broaden the pulse width and reduce the peak power before the pulse amplification, thereby reducing the nonlinear effect in the amplification process. The optimized seed light is amplified by an erbium-doped optical fiber amplifier (EDFA) and then pumped into a 10-m-long single-mode ZBLAN fiber which has a core/clad diameter of $6 / 125 \mu \mathrm{m}$, a numerical aperture (NA) of 0.2 , and a cut-off wavelength at $1650 \mathrm{~nm}$. The fitted dispersion profile of the ZBLAN fiber is shown in Fig. 2. The fiber has a zero-dispersion wavelength near $1700 \mathrm{~nm}$, and the dispersion is relatively flat over the wavelength of $1550 \mathrm{~nm}$ $2000 \mathrm{~nm}$. A measurement of the fiber attenuation is provided by FiberLabs Inc., in $1000 \mathrm{~nm}-3000 \mathrm{~nm}$, and the fiber exhibits losses below $0.1 \mathrm{~dB} / \mathrm{m}$. The performance of the optical spectrum is observed by an optical spectral analyzer (YOKOGAWA, AQ6375) with a resolution of $0.2 \mathrm{~nm}$. The pulse width and the optical pulse trains are measured by a commercial autocorrelator (Femtochrome, FR-103HS) and oscilloscope (Tektronix, MSO4104), respectively. 


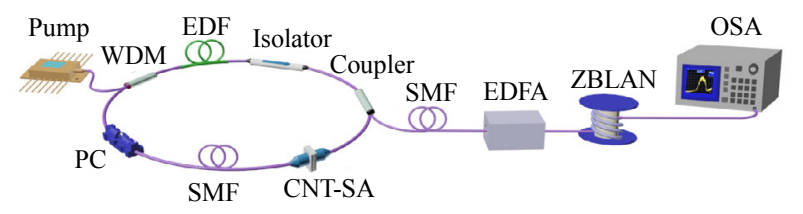

Fig. 1 Experimental setup of spectrally flat supercontinuum generation pumped by the mode-locked laser. CNT-SA: carbon-nanotube-based saturable absorber; WDM: wavelength division multiplexing; PC: polarization controller; SMF: single-mode fiber; EDF: erbium-doped fiber; EDFA: erbium-doped fiber amplifier; OSA: optical spectrum analyzer.

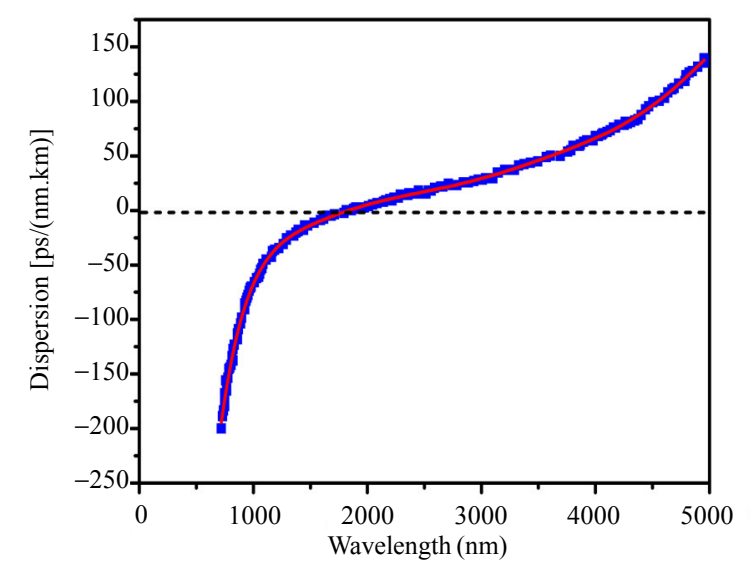
fiber.

Fig. 2 Fitted dispersion data of the single-mode ZBLAN

\section{Results and discussion}

The stable mode-locked condition of the seed laser is achieved at an input pump power of $72.6 \mathrm{~mW}$, and the average output power of the laser is about $804 \mu \mathrm{W}$. The corresponding peak power is $\sim 37.6 \mathrm{~W}$, and the time-bandwidth product is estimated to be $\sim 0.76$. Figure 3(a) depicts the time-domain pulse and frequency-domain spectrum of the seed laser. The blue curve presents the mode-locked optical spectrum of the seed fiber laser, and the $3-\mathrm{dB}$ spectral bandwidth is about $5.3 \mathrm{~nm}$ centered at $1562.4 \mathrm{~nm}$. The red curve shows the autocorrelation trace fitted by $\operatorname{sech}^{2}$-shaped pulse, and the pulse width is calculated to be $\sim 1.16$ ps. The corresponding radio frequency and oscilloscope trace are shown in Fig.3(b). The output pulse train has a repetition rate of $18.44 \mathrm{MHz}$, and the signal-to-noise ratio (SNR) is $\sim 45 \mathrm{~dB}$ at the fundamental frequency of $18.44 \mathrm{MHz}$.

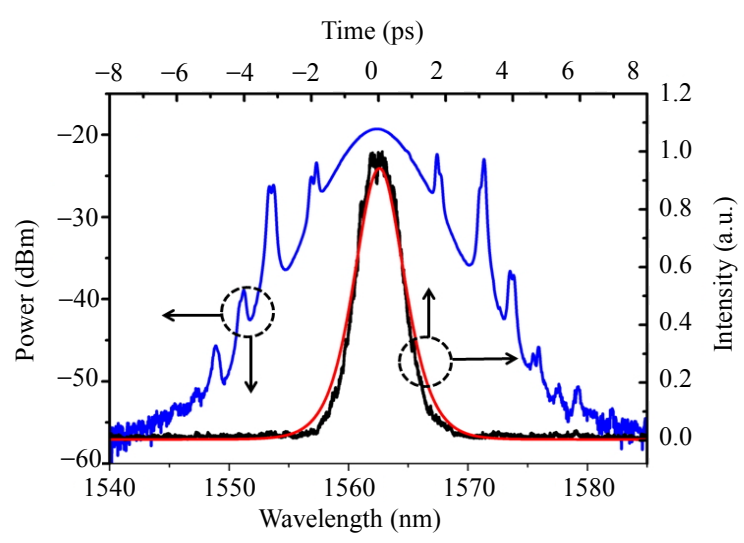

(a)

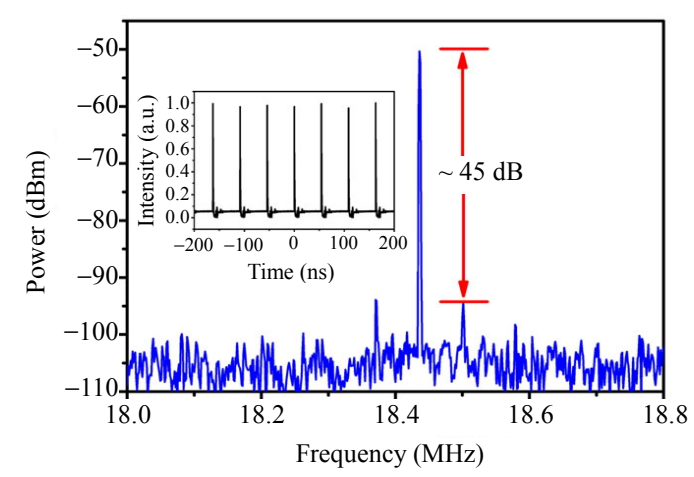

(b)

Fig. 3 Time and frequency domain characteristics of the seed laser: (a) mode-locked optical spectrum (blue curve) and autocorrelation trace of pulse and $\mathrm{sech}^{2}$-shape pulse fitting (red curve) and (b) corresponding RF spectrum, the insert picture is the output pulse train.

Figure 4 shows the output spectra of SC after the ZBLAN fiber at different power levels. As the input average power increases, the spectrum gradually broadens to the longer wavelength. When the average output power $\left(P_{\mathrm{av}}\right)$ of $\mathrm{SC}$ is $0.61 \mathrm{~mW}$ corresponding to the input pulse with the peak power of $99 \mathrm{~W}$, the spectral broadening is not obvious, and the $20-\mathrm{dB}$ bandwidth is about $30 \mathrm{~nm}$. When $P_{\text {av }}$ of SC is $5.25 \mathrm{~mW}$ corresponding to the input pulse with the peak power of $934 \mathrm{~W}$, the spectrum only broadens to $1800 \mathrm{~nm}$, and the $20-\mathrm{dB}$ bandwidth is about $185 \mathrm{~nm}$. When $P_{\text {av }}$ increases to $52.5 \mathrm{~mW}$ (the power is limited by our existing equipment), at this point, the pulse split, the long wavelength side of the $\mathrm{SC}$ extends to $2000 \mathrm{~nm}$, and the $20-\mathrm{dB}$ bandwidth is about $290 \mathrm{~nm}$. However, all the spectra show relatively large fluctuation over the 
wavelength ranging from $1600 \mathrm{~nm}$ to $1800 \mathrm{~nm}$.

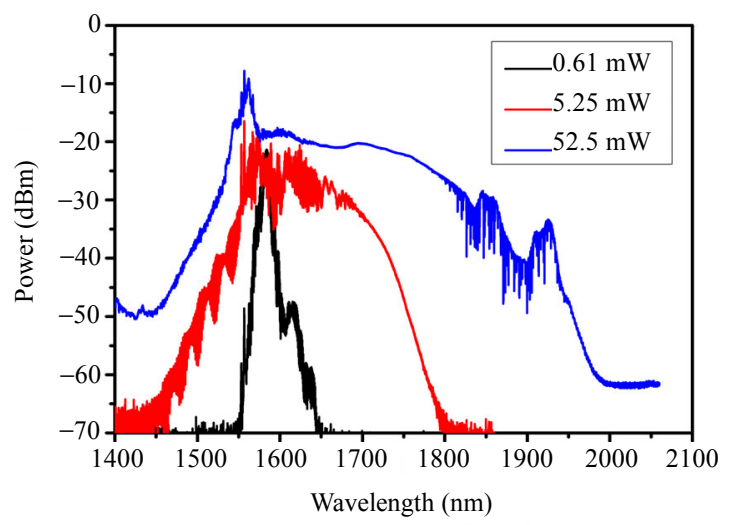

Fig. 4 Spectral distribution after the ZBLAN fiber for different average output powers.

In order to improve the spectral flatness, the SMF with the lengths of $0 \mathrm{~m}, 50 \mathrm{~m}, 100 \mathrm{~m}, 150 \mathrm{~m}$, as well as $200 \mathrm{~m}$, is connected to the output of the mode-locked fiber laser, respectively. The tendency of the pulse width and peak power varying with the access length of the SMF is presented in Fig. 5, the inset picture of Fig. 5 shows the autocorrelation trace after the 200-m-long SMF fitted by Gauss-shaped pulse, and the pulse width is calculated to be $\sim 23.7$ ps. When the access length of the SMF is less than $200 \mathrm{~m}$, as the length of the SMF $\left(L_{\mathrm{smf}}\right)$ increases, the pulse width increases, and the pulse is chirped, so that the peak power lowers. When $L_{\text {smf }}$ extends to $210 \mathrm{~m}$, we observe that the pulse width and the spectral bandwidth are all slightly narrowed over the repeated experiments. Such changes of pulse in the time and frequency domains are believed to be originated from the excess loss of the increased number of fiber connectors. So, the access length of the SMF is optimized at $200 \mathrm{~m}$ in our experiment.

The pulses after passing the SMF are then launched into the EDFA. In the experiment, in order to verify the role of the SMF, the chirped pulse is firstly amplified at an average power of tens of watts to avoid the pulse fission. Figure 6(a) shows the pulse width and the pulse energy, as well as the peak power varying with the access length of the SMF after the pulse amplification. Under the same setting of EDFA, as the length of the SMF increases, the amplified pulse energy increases, indicating that the conversion efficiency of the amplifier is improved. In the meantime, the peak power of the pulses decreases due to the pulse broadening during the amplification process. Figure 6(b) shows the measured autocorrelation trace when $L_{\mathrm{smf}}$ is $200 \mathrm{~m}$. The pulse width is calculated to be $\sim 24.5 \mathrm{ps}$ fitted by the Gauss-shaped pulse, which is slightly larger than the measured pulse width of $\sim 23.7 \mathrm{ps}$ before the pulse amplification. The time-bandwidth product of the pulse with the SMF of $200 \mathrm{~m}$ is calculated to be $\sim 12$ when the $3-\mathrm{dB}$ spectral bandwidth is measured to be $\sim 4 \mathrm{~nm}$, such highly chirped pulse with improved pulse energy could be further potentially compressed for obtaining the higher peak power. By further increasing the output average power from EDFA, although single pulse train can be observed in the oscilloscope, the autocorrelation trace shows multiple peaks, indicating that the pulse fission exists at a high power level.

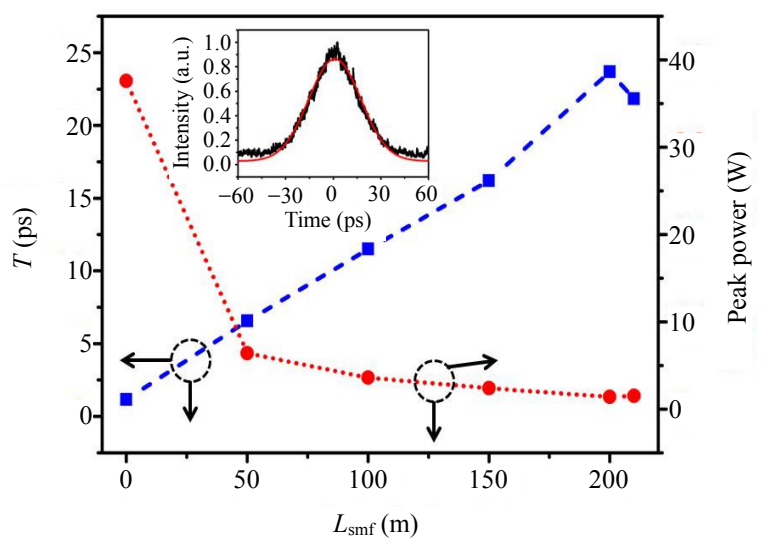

Fig. 5 Tendency of pulse width (blue curve) and peak power (red curve) varying with the access length of the SMF before the pulse amplification. Insert: the autocorrelation trace after the 200-m-long SMF fitted by the Gauss-shaped pulse.

Figure 7 shows the SC spectrum exhibiting asymmetric broadening with the SMF of $200 \mathrm{~m}$, and we achieve the spectral fluctuation less than $1.29 \mathrm{~dB}$ over $1571.8 \mathrm{~nm}-1803.1 \mathrm{~nm}$. In contrast, when the SMF is not connected as shown in the inset of Fig. 7, the fluctuation of the optical spectrum is about $8.30 \mathrm{~dB}$ between $1571.8 \mathrm{~nm}$ and $1803.1 \mathrm{~nm}$. When a 50-m-long SMF is connected, the fluctuation of the 
optical spectrum is about $4.93 \mathrm{~dB}$ between $1571.8 \mathrm{~nm}$ and $1803.1 \mathrm{~nm}$. When a 100-m-long SMF is connected, the spectral fluctuation turns to $2.63 \mathrm{~dB}$ in the interval aforementioned. The recent work, a compact broadband $\mathrm{SC}$ generation in a single-mode ZBLAN fiber pumped by femtosecond pulses from an erbium-doped fiber laser, shows the SC spectrum spanning from $1100 \mathrm{~nm}$ to $3200 \mathrm{~nm}$ with a spectral fluctuation less than $8 \mathrm{~dB}$ [18]. Differently, we have achieved the SC generation with the spectral fluctuation less than $1.29 \mathrm{~dB}$ over $1571.8 \mathrm{~nm}$ $1803.1 \mathrm{~nm}$. The results indicate that optimizing the pulse width before the amplifier is of great significance for improving the spectral flatness.

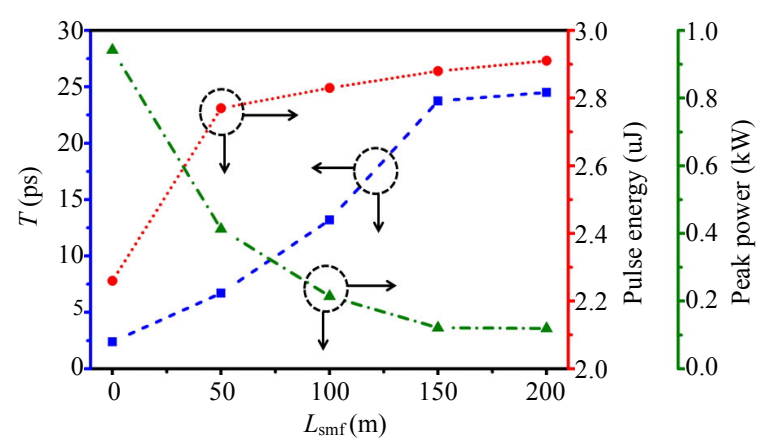

(a)

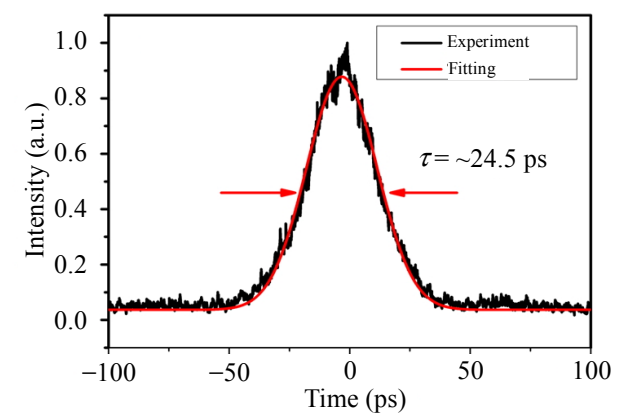

(b)

Fig. 6 Pulse characteristics after EDFA: (a) pulse width (blue curve), pulse energy (red curve), and peak power (green curve) varying with the access length of the SMF after the pulse amplification and (b) autocorrelation trace fitted by the Gauss-shaped pulse after EDFA when $L_{\mathrm{smf}}$ is $200 \mathrm{~m}$.

Note that as the length of the SMF increases, the flatness of the optical spectrum is significantly improved. As the length of the SMF increases, the pulse width increases. During the whole process of pulse width broadening, the repetition rate remains unchanged, and the output power of the tail end of the SMF is reduced, causing a drop in the peak power. This method is used to suppress nonlinear effects in the amplification process to minimize pulse fission. When such amplified pulse train pumps a highly nonlinear fiber in the normal dispersion region, it is possible to generate an SC spectrum with better flatness and stability [19]. Previous works on numerical simulations of SC based on the generalized nonlinear Schrödinger equations have been performed [20-22], in which the combination of dispersion and various nonlinear effects has been taken into account. It is found that the self-phase modulation is the primary cause of spectral broadening in the first stage when the fiber is pumped by the pulses in the normal dispersion region [23]. When the pulses further propagate along the nonlinear medium, most of the pulse energy is shifted toward the long wavelength side due to the nonlinearity of Raman soliton self-frequency shifting [24], resulting in a broadband SC spectrum.

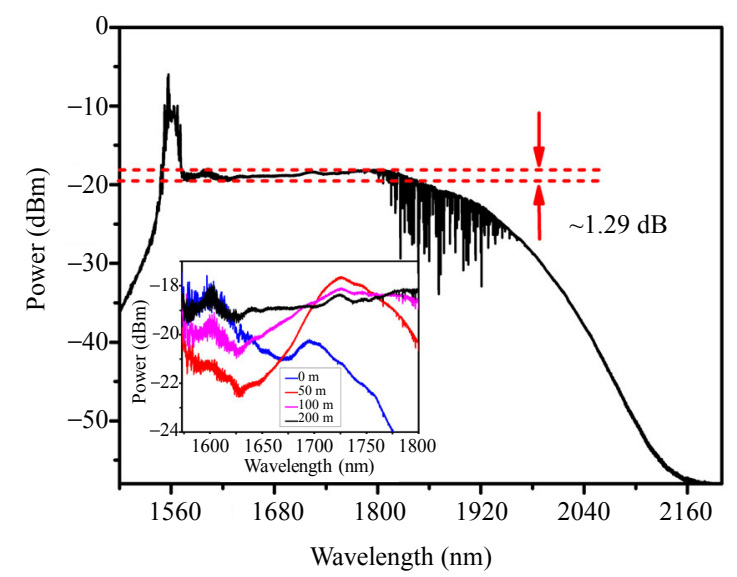

Fig. 7 Spectral distribution after the ZBLAN fiber when a 200-m-long SMF is connected. Insert: the comparison of spectral distribution when different lengths of the SMF are connected.

\section{Conclusions}

We have experimentally demonstrated a flat supercontinuum generation in a 10 -m-long ZBLAN fiber pumped by CNT-SA passively mode-locked fiber laser. In order to improve the spectral flatness of $\mathrm{SC}$, the SMF with different lengths is connected to the output of the mode-locked fiber laser. It is 
found that optimizing the pulse width and peak power of the seed source can improve the flatness of the optical spectrum. The output SC spectrum exhibiting the best spectral flatness with the fluctuation less than $1.29 \mathrm{~dB}$ in the range of $1571.8 \mathrm{~nm}$ to $1803.1 \mathrm{~nm}$ has been achieved. Since SC sources possess the good beam quality and high spectral stability, they have potential applications in various long-distance optical sensing.

\section{Acknowledgment}

This work was supported by the National Natural Science Foundation of China (Grant Nos. 61605108, 61635006, and 61735009) and Shanghai Young Oriental Scholar 2016 (Grant No. QD2016025).

Open Access This article is distributed under the terms of the Creative Commons Attribution 4.0 International License (http://creativecommons.org/licenses/by/4.0/), which permits unrestricted use, distribution, and reproduction in any medium, provided you give appropriate credit to the original author(s) and the source, provide a link to the Creative Commons license, and indicate if changes were made.

\section{References}

[1] S. Lambert-Girard, M. Allard, M. Piché, and F. Babin, "Differential optical absorption spectroscopy lidar for mid-infrared gaseous measurements," Applied Optics, 2015, 54(7): 1647-1656.

[2] C. R. Petersen, U. Møller, I. Kubat, B. B. Zhou, S. Dupont, J. Ramsay, T. Benson, et al., "Mid-infrared supercontinuum covering the $1.4-13.3 \mu \mathrm{m}$ molecular fingerprint region using ultra-high NA chalcogenide step-index fibre," Nature Photonics, 2014, 8(11): 830-834.

[3] A. Mukherjee, S. V. D. Porten, and C. K. N. Patel, "Standoff detection of explosive substances at distances of up to $150 \mathrm{~m}$," Applied Optics, 2010, 49(11): 2072-2078.

[4] B. Takashi, N. C. Conley, and S. W. Choi, "Multispectral photoacoustic microscopy of lipids using a pulsed supercontinuum laser," Biomedical Optics Express, 2018, 9(1): 276-288.

[5] V. V. Alexander, Z. N. Shi, M. N. Islam, K. Ke, G. Kalinchenko, M. J. Freeman, et al., "Field trial of active remote sensing using a high-power short-wave infrared supercontinuum laser," Applied Optics, 2013,
52(27): 6813-6823.

[6] C. Lin and R. H. Stolen, "New nanosecond continuum for excited-state spectroscopy," Applied Physics Letters, 1976, 28(4): 216 218.

[7] M. Michalska, J. Mikolajczyk, J. Woijas, and J. Swiderski, "Mid-infrared, super-flat, supercontinuum generation covering the $2-5 \mu \mathrm{m}$ spectral band using a fluoroindate fibre pumped with picosecond pulses," Scientific Reports, 2016, 6: 39138-1-39138-6.

[8] L. Y. Yang, B. Zhang, K. Yin, T. Y. Wu, Y. J. Zhao, and J. Hou, "Spectrally flat supercontinuum generation in a holmium-doped ZBLAN fiber with record power ratio beyond $3 \mu \mathrm{m}$," Photonics Research, 2018, 6(5): 417-421.

[9] J. C. Gauthier, V. Fortin, J. Y. Carrée, S. Poulain, M. Poulain, R. Vallée, et al., "Mid-IR supercontinuum from 2.4 to $5.4 \mu \mathrm{m}$ in a low-loss fluoroindate fiber," Optics Letters, 2016, 41(8): 1756-1759.

[10] K. Liu, J. Liu, H. X. Shi, F. Z. Tan, and P. Wang, "High power mid-infrared supercontinuum generation in a single-mode ZBLAN fiber with up to $21.8 \mathrm{~W}$ average output power," Optics Express, 2014, 22(20): 24384-24391.

[11] U. Møller, Y. Yu, L. Kubat, C. R. Petersen, X. Gai, L.Brilland, D.Méchin, et al., "Multi-milliwatt mid-infrared supercontinuum generation in a suspended core chalcogenide fiber," Optics Express, 2015, 23(3): 3282-3291.

[12] F. Théberge, N. Bérubé, S. Poulain, S. Cozic, L. R. Robichaud, M. Bernier, et al., "Watt-level and spectrally flat mid-infrared supercontinuum in fluoroindate fibers," Photonics Research, 2018, 6(6): 609-613.

[13] W. Q. Yang, B. Zhang, K. Yin, X. F. Zhou, and J. Hou, "High power all fiber mid-IR supercontinuum generation in a ZBLAN fiber pumped by a $2 \mu \mathrm{m}$ MOPA system," Optics Express, 2013, 21(17): 19732-19742.

[14] X. Jiang, N. Y. Joly, M. A. Finger, F. Babic, G. K. L. Wong, J. C. Travers, et al., "Deep-ultraviolet to mid-infrared supercontinuum generated in solid-core ZBLAN photonic crystal fibre," Nature Photonics, 2015, 9(2): 133-139.

[15] G. S. Qin, X. Yan, C. Kito C, M. S. Liao, C. Chaudhari, T. Suzuki, et al., "Ultrabroadband supercontinuum generation from ultraviolet to $6.28 \mu \mathrm{m}$ in a fluoride fiber," Applied Physics Letters, 2009, 95(16): 161103-1-161103-3.

[16] T. Liang and X. M. Feng, "Research progress toward flat supercontinuum generation in fibers," Laser \& Optoelectronics Progress, 2016, 53(6): 10-28.

[17] L. Y. Shi L, L. A. Sordillo, A. Rodríguez-Contreras, and R. R. Alfano, "Transmission in near- 
infrared optical windows for deep brain imaging," Journal of Biophotonics, 2016, 9(1-2): 38-43.

[18] S. R. Yemineni, W. J. Lai, A. Alphones, and P. Shum, "Mid-IR supercontinuum generation in a single-mode ZBLAN fiber by erbium-doped fiber laser," Optical Engineering, 2018, 57(11): 111804-1 $-111804-5$.

[19] A. M. Heidt, "Pulse preserving flat-top supercontinuum generation in all-normal dispersion photonic crystal fibers," Journal of the Optical Society of America B Optical Physics, 2010, 27(3): $550-559$.

[20] J. M. Dudley, G. Genty, and S. Coen, "Supercontinuum generation in photonic crystal fiber," Reviews of Modern Physics, 2006, 78(4): 1135-1184.

[21] L. Liu, G. S. Qin, Q. J. Tian, D. Zhao, and W. P. Qin, "Numerical investigation of mid-infrared super- continuum generation up to $5 \mu \mathrm{m}$ in single mode fluoride fiber," Optics Express, 2011, 19(11): 10041-10048.

[22] C. Agger, C. Petersen, S. Dupont, H. Steffensen, J. K. Lyngsø, C. L. Thomsen, et al., "Supercontinuum generation in ZBLAN fibers-detailed comparison between measurement and simulation," Journal of the Optical Society of America B Optical Physics, 2012, 29(4): 635-645.

[23] J. Swiderski, "High-power mid-infrared supercontinuum sources: Current status and future perspectives," Progress in Quantum Electronics, 2014, 38(5): 189-235.

[24] K. Yin, B. Zhang, L. Y. Yang, and J. Hou, " $15.2 \mathrm{~W}$ spectrally flat all-fiber supercontinuum laser source with $>1 \mathrm{~W}$ power beyond $3.8 \mu \mathrm{m}$," Optics Letters, 2017, 42(12): 2334-2337. 